\title{
Transient Rates of Synthesis of Individual Polypeptides in E. coli Following Temperature Shifts
}

\author{
Peggy G. Lemaux, ${ }^{*}$ Sherrie L. Herendeen, Philip \\ L. Bloch and Frederick C. Neidhardt \\ Departmerll of Microbiology \\ The University of Michigan \\ Ann Arbor, Michigan 48109
}

\section{Summary}

The effect of temperature shifts on the rates of synthesis of selected individual polypeptides in wild-type E. coli was studied through puise-labeling and resolution of protein by the O'Farrell twodimensional gel system.

The overall rate of protein synthesis quickly accelerated to its new steady state rate after temperature shifts, but the differential rates of synthesis of most individual polypeptides studied either increased transiently (as much as $\mathbf{5 0}$ fold) or decreased transiently (as much as 10 fold). Their new steady state values, usually the preshift values, were reached $20 \mathrm{~min}$ after the shift. The magnitude of the responses depended upon the size of the temperature shift, and a shift downward generated responses opposite to those of a shift upward. The ten aminoacyl-tRNA synthetases and five transcriptional or other translational factors studied responded with greater similarity than did a group of nine unidentified polypeptides chosen from different regulatory classes. Guanosine tetraphosphate accumulated transiently upon a temperature shift upward, but reducing this accumulation did not in general alter the quantitative responses of the polypeptides.

For some proteins, these transients accomplish rapid attainment of a new steady state level. Many of the transients seem to be active regulatory responses.

\section{Introduction}

Living systems are under selective pressure to regulate a vast array of biochemical reactions during frequently changing environmental conditions. For example, enteric bacteria synthesize cellular components approximately 4 times faster at $39^{\circ} \mathrm{C}$ than at $20^{\circ} \mathrm{C}$. Considering the perhaps 2000 individual chemical reactions involved in growth, it would seem that the relationship between temperature and cellular functions must be quite complex. Two simple generalizations, however, seem to hold. One is that the relative amounts of major cellular components and macromolecules remain reasonably constant over this temperature range. Schaechter, Maaløe and Kjeldgaard (1958), for example,

* Present address: Departments of Medicine and Genetics, Stanford University School of Medicine, Stanford, California 94305. showed that cell volume, mass, FINA, DNA and the number of nuclei per cell in Salmonella typhimurium were nearly constant for a given medium at 37 and $25^{\circ} \mathrm{C}$, despite the 2 fold faster growth rate at $37^{\circ} \mathrm{C}$. The relative constancy of these parameters with respect to each other therefore suggests that these components have nearly equal temperature characteristics - that is, that their rates of function are affected approximately to the same extent by temperature.

The second generalization is that over this tem perature range, the rate of cell growth can be described by the same relationship developed by Arrhenius to describe the temperature dependence of individual, simple chemical reactions (Ingraham and Marr, 1963). This fact has never been fully accounted for by cell theory. The integration of the myriad reactions of a cell into a single parameter (the growth rate), which can be described by a relationship developed for a single reaction, is usualiy attributed either to some one "master reaction" being growth rate-limiting over this range $(\mathrm{Ng}$, Ingraham and Marr, 1962) or to the numerous separate reactions in the cell being so integrated by control devices that individual reactions cannot be rate-limiting, and thus cannot generate the kind of complex temperature-dependence that would be expected if first one and then another reaction became rate-limiting with progressive changes in temperature. It is not clear to what extent enzyme repression, as distinct from enzyme inhibition, might be involved in such integration.

Several previous studies have probed the effects of extremes of temperature, either high (Patterson and Gillespie, 1972) or low (Ingraham and Marr, 1963), on a variety of cellular processes. The use in our laboratory of mutants with various temperature-sensitive components, however, necessitated the examination of the synthesis of individual polypeptides in the wild-type strain subjected to temperature shifts well within its normal range of growth temperatures. The results reported here indicate that constancy of the differential rates of synthesis of many individual polypeptides in cells in steady state growth at various temperatures is achieved only after dramatic transient changes which take place following a temperature shift. In many cases, these changes are of sufficient magnitude that they must be taken into account when interpreting the behavior of these proteins in temperature-sensitive mutant cells.

\section{Results}

Effect of Temperature on the Growth of E. coli Strain NC3

Cultures of strain NC3 were grown in steady state either at $28^{\circ} \mathrm{C}$ and then shifted up to $33,36,39$ and 
$42^{\circ} \mathrm{C}$, or grown at $36^{\circ} \mathrm{C}$ and then shifted down to $28^{\circ} \mathrm{C}$. In each case, the steady state growth rate characteristic of the new temperature was attained within $2 \mathrm{~min}$. As has been shown for other $\mathrm{E}$. coli strains (Ingraham and Marr, 1963), a plot of the log of the specific growth rate constant $(k)$ as a function of the inverse of absolute temperature is linear between 28 and $39^{\circ} \mathrm{C}$ (slope: $12,700 \mathrm{cal} / \mathrm{mol}$ ). In our strain, the growth rate is the same at 42 as at $39^{\circ} \mathrm{C}$.

\section{Effect of a Temperature Shift-up on the Relative Differential Rates of Synthesis of Individual Polypeptides}

Individual polypeptides exhibited transient fluctuations in their differential rates of synthesis (rate of synthesis of that protein relative to the rate of synthesis of total protein) following a temperature shift-up of $11^{\circ} \mathrm{C}$. After these transient changes, in most cases, the rates for the polypeptides assumed their preshift rates. Table 1 contains the results for a number of individual polypeptides selected to depict representative behavior. The data in Table 1 indicate the sensitivity of the method in detecting such changes and the small magnitude of error in these measurements. For most polypeptides, the transient response was detectable at the first sampling time after the shift ( $3 \mathrm{~min})$, and the maximum response occurred within the first $10 \mathrm{~min}$. In many cases, the differential rate of synthesis returned to near the preshift rate by 25-45 min after the shift. Measurements of the relative level of individual polypeptides (T. Slykhouse, unpublished results) in cells in steady state growth at 30 and $37^{\circ} \mathrm{C}$ confirmed that the differential rates of synthesis, although the same for most polypeptides, were different for such polypeptides as B56.5, C137 and F84.1.

The results from temperature shifts of varying magnitudes for 26 different polypeptides are displayed in Figures 1, 2 and 3.

Eleven aminoacyl-tRNA synthetase polypeptides are grouped in Figure 1 (both subunits are shown for phenylalanyl-tRNA synthetase). A common response pattern can be seen. The relative differential rate of synthesis of each declined rapidly upon a temperature shift from 28 to $42^{\circ} \mathrm{C}$. Temperature shifts from $28^{\circ} \mathrm{C}$ to 39,36 and $33^{\circ} \mathrm{C}$ produced progressively diminishing responses, which in the case of five synthetases (arginyl-, aspartyl-, glycyl-, isoleucyl- and leucyl-tRNA synthetases) actually became stimulations of synthesis. All the synthetases appeared to be recovering from the transient effect of the temperature shift by $10 \mathrm{~min}$, irrespective of the magnitude of that shift.

The results for the protein synthesis elongation

\begin{tabular}{|c|c|c|c|c|c|c|}
\hline $\begin{array}{l}\text { Time } \\
\text { after } \\
\text { Shift } \\
\text { (min) }\end{array}$ & GlnRs & $\begin{array}{l}\text { PheRs } \\
\alpha \text { Subunit }\end{array}$ & $E F-G$ & s1 & $\mathrm{C} 137^{\mathrm{b}}$ & F84.1 \\
\hline 0 & 1.00 & 1.00 & 1.00 & 1.00 & 1.00 & 1.00 \\
\hline 3 & $0.82 \pm 0.03^{\mathrm{c}}$ & $0.59 \pm 0.08$ & $0.73 \pm 0.04$ & $0.48 \pm 0.04$ & $0.53 \pm 0.05$ & $21.67 \pm 1.26$ \\
\hline 7 & $0.69 \pm 0.04$ & $0.57 \pm 0.04$ & $0.47 \pm 0.01$ & $0.77 \pm 0.02$ & $0.52 \pm 0.02$ & $35.74 \pm 1.78$ \\
\hline 10 & $0.82 \pm 0.04$ & $0.71 \pm 0.03$ & $0.57 \pm 0.01$ & $0.87 \pm 0.03$ & $0.59 \pm 0.04$ & $14.50 \pm 1.78$ \\
\hline 15 & $0.91 \pm 0.06$ & $0.98 \pm 0.05$ & $0.77 \pm 0.02$ & $0.86 \pm 0.05$ & $0.64 \pm 0.05$ & $5.50 \pm 0.68$ \\
\hline 25 & $0.96 \pm 0.04$ & $1.18 \pm 0.03$ & $0.78 \pm 0.01$ & $0.82 \pm 0.06$ & $0.69 \pm 0.06$ & 4.89 \\
\hline 45 & $0.99 \pm 0.04$ & $0.94 \pm 0.14$ & $0.78 \pm 0.03$ & $0.90 \pm 0.05$ & 0.66 & $5.60 \pm 0.10$ \\
\hline
\end{tabular}

${ }^{a}$ A culture of $\mathrm{E}$. coli strain NC3 was grown in fully synthetic rich medium, lacking isoleucine, leucine and valine, and labeled with ${ }^{14} \mathrm{C}$ arginine and-lysine during steady state growth at $28^{\circ} \mathrm{C}$. Incorporation was stopped by the addition of unlabeled amino acids, and portions of the culture were pulse-labeled with ${ }^{3} \mathrm{H}$-isoleucine, -leucine and -valine at various times before and after the shift to $42^{\circ} \mathrm{C}$ (see Experimental Procedures). The ${ }^{3} \mathrm{H} /{ }^{14} \mathrm{C}$ ratio of individual polypeptides was determined and divided by the ${ }^{3} \mathrm{H} /{ }^{14} \mathrm{C}$ ratio of total protein; this number represents the differential rate of synthesis of the polypeptide. The differential rate of synthesis of a polypeptide after the shift to $42^{\circ} \mathrm{C}$ was divided by the average of two determinations of the differential rate of synthesis of that same polypeptide made under steady state conditions at $28^{\circ} \mathrm{C}$. This number is referred to as the relative differential rate of synthesis and is thus defined as being equal to 1.0 prior to the shift. Two independent determinations of the relative differential rate of synthesis from each extract were made for each polypeptide taken at a given time. These two values were then averaged.

${ }^{b}$ Abbreviations are as follows: GInRS, glutaminyl-tRNA synthetase; PheRS, phenylalanyltRNA synthetase; EF-G, elongation factor G; Si, ribosomal protein (30S subunit) S1. The alphanumeric designations (S. Pedersen, P. L. Bloch, S. Reeh and F. C. Neidhardt, manuscript in preparation) of polypeptides are based on the position of the polypeptide in the O'Farrell two-dimensional acrylamide gel system (O'Farrell, 1975). The numeric portion refers to the apparent molecular weight of the polypeptide (F84.1-molecular weight $84.1 \times 10^{3}$ daltons) as determined from the migration of the polypeptide from the origin in the second dimension. The letter prefix is bascd on the position of the polypeptide in the first dimension which was arbitrarily divided into six segments, A-F, with $A$ containing those polypeptides having the most acid isoelectric points.

${ }^{c}$ Standard error of two measurements made on the same extract. 


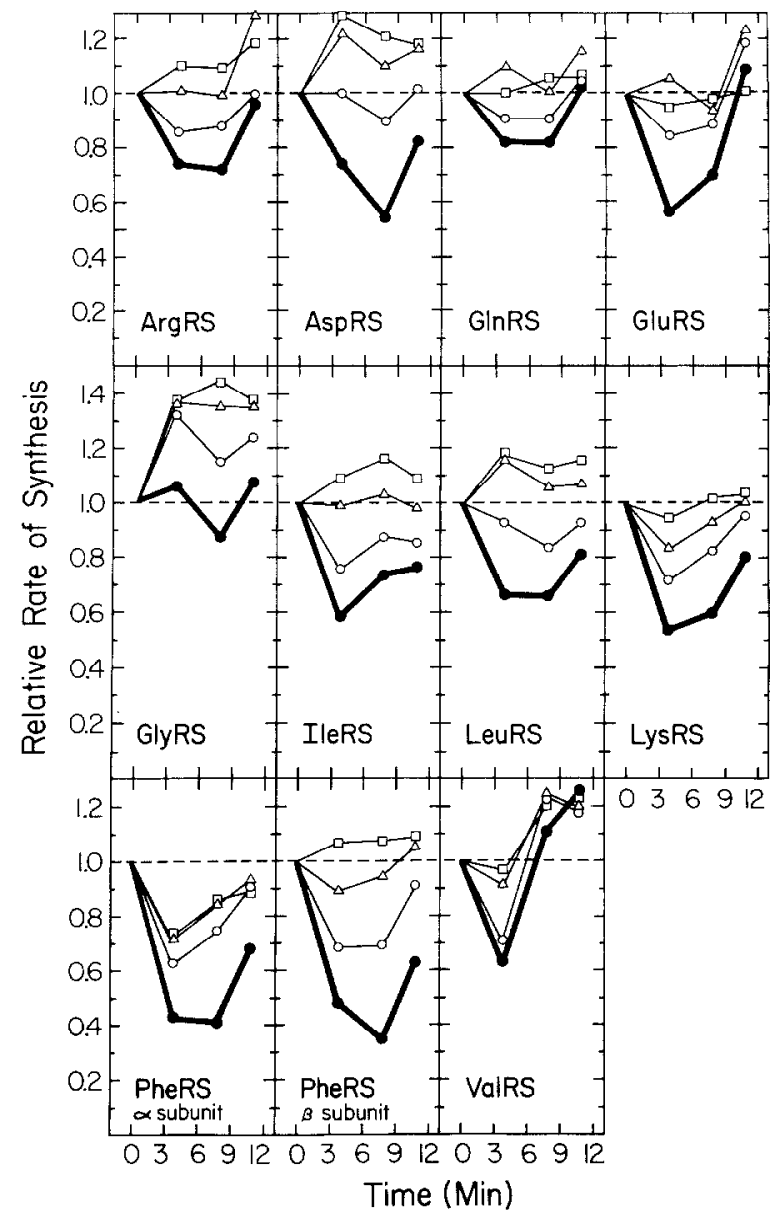

Figure 1. Relative Differential Rates of Synthesis of Individual Aminoacyl-tRNA Synthetase Polypeptides Following Temperature Shifts-up of E. coli Strain NC3

A culture of strain NC3 was grown in fully synthetic rich medium minus isoleucine, leucine and valine in steady state. A cuilture was grown at $28^{\circ} \mathrm{C}\left(\mathrm{k}, 0.89 \mathrm{hr}{ }^{1}\right)$ and split into four culture flasks, and these were then shifted to $42^{\circ} \mathrm{C}\left(\mathrm{k}, 1.93 \mathrm{hr}^{-1}\right), 39^{\circ} \mathrm{C}\left(\mathrm{k}, 1.89 \mathrm{hr}^{-1}\right)$, $36^{\circ} \mathrm{C}\left(\mathrm{k}, 1.54 \mathrm{hr}^{-1}\right)$ and $33^{\circ} \mathrm{C}\left(\mathrm{k}, 1.30 \mathrm{hr}^{-1}\right)$. The temperature shifts of all tour cultures were carried out at the same time and labeled as described in Table 1 . The heavy line represents the results caused by the shift from 28 to $42^{\circ} \mathrm{C}$. The abbreviations used for the aminoacyl-tRNA synthetases are designated by the accepted three-letter abbreviation for the amino acid, combined with RS for tRNA synthetase-for example, ArgRS for arginyl-tRNA synthetase and so on (Neidhardt, McKeever and Parker, 1975). $(-)$ ) shift from 28 to $42^{\circ} \mathrm{C} ;(\mathrm{O}-\mathrm{O})$ shift from 28 to $39^{\circ} \mathrm{C}$; $\left(\triangle \square \triangle\right.$ ) shift from 28 to $36^{\circ} \mathrm{C}$; and $(\square \square \square)$ shift from 28 to $33^{\circ} \mathrm{C}$.

factors $G(E F-G)$, Ts (EF-Ts) and Tu (EF-Tu), ribosomal protein $\mathrm{S} 1$, and RNA polymerase subunits $\alpha$ and $\beta$ are presented in Figure 2. In general, the responses of these polypeptides closely resembled those of the synthetases, with the exception of EFTu. This polypeptide exhibited the least fluctuation in differential rate, and the usual ordered effect of increasing temperature seemed not to hold. Although standard errors for the measurements are

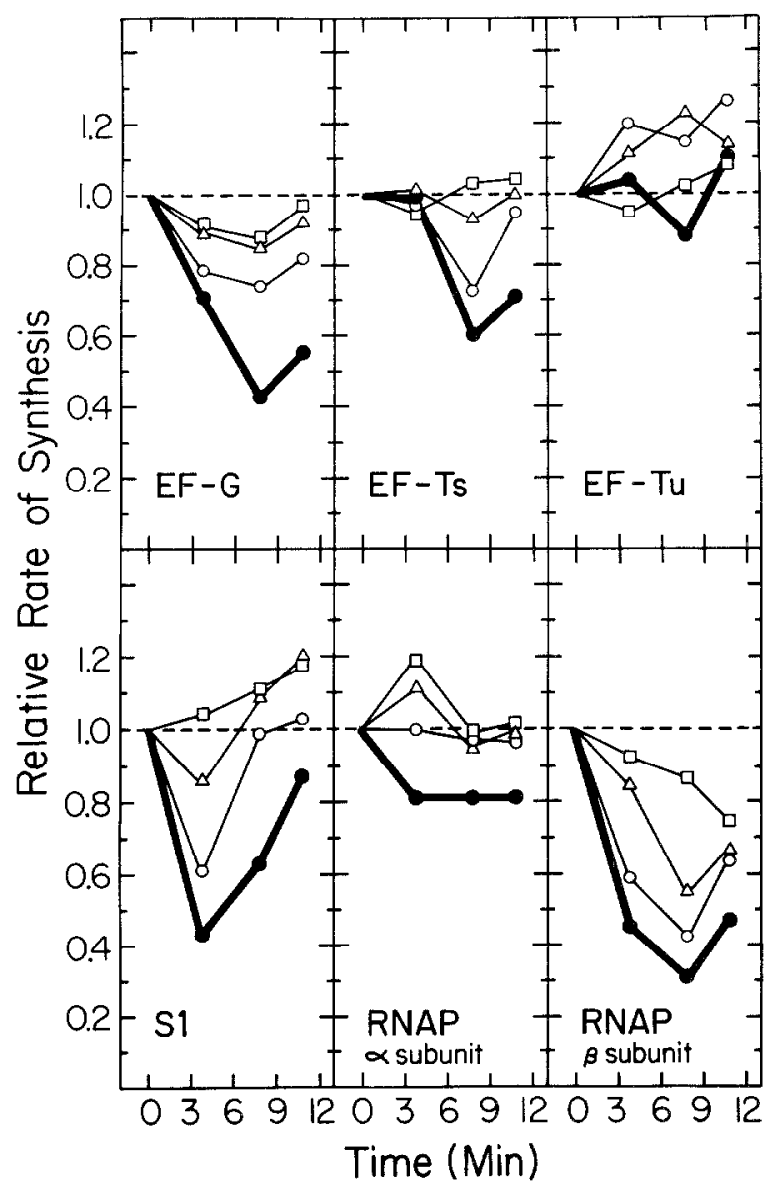

Figure 2. Relative Differential Rates of Synthesis of Individual, Identified Transcriptional and Other Translational Polypeptides Following Temperature Shifts-up of E. coli Strain NC3

Cells were grown as described in Figure 1 and labeled, and the calculations were made as described in Table 1. Symbols are as described in Figure 1. The abbreviations are as follows: EF-G, elongation factor $G$; EF-Ts, elongation factor Ts; EF-Tu, elongation factor Tu; $S 1$, ribosomal protein (30S subunit) S1; RNAP. RNA polymerase.

not shown on these graphs, the magnitude of the errors indicates that the differences among the responses at the various temperatures are significant.

In Figure 3, unidentified polypeptides were selected to illustrate the variety of responses found for polypeptides in the cell (note change of ordinate scales). This selection included examples of polypeptides that exhibit different regulatory patterns in response to a variety of growth media (S. Pedersen, P. L. Bloch, S. Reeh and F. C. Neidhardt, manuscript in preparation). Unlike the homogeneity of the responses for the functionally related polypeptides in Figures 1 and 2, these polypeptides exhibited several different behaviors. Four of the polypeptides (B56.5, F24.5, F84.1 and G32.8) showed increases in their relative differen- 


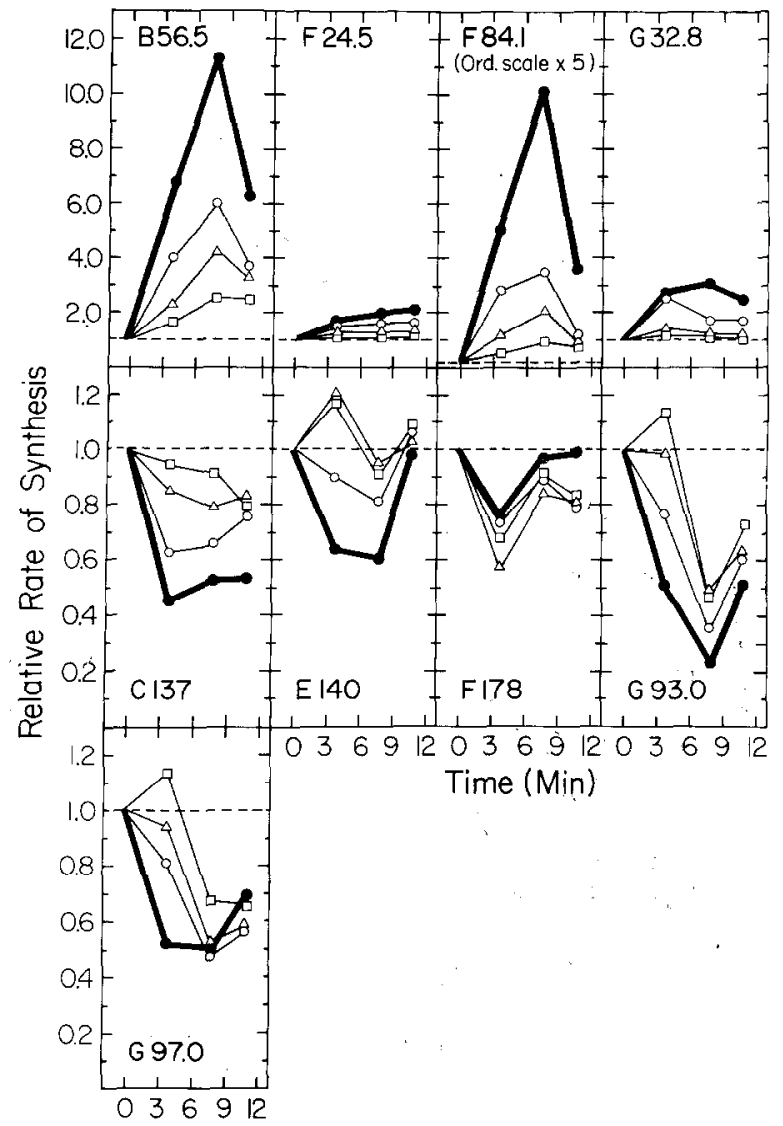

Figure 3. Relative Differential Rates of Synthesis of Unidentified Polypeptides Following Temperature Shifts-up of E. coli Strain NC3

Cells were grown as described in Figure 1 and labeled, and the calculations were made as described in Table 1. Symbols are as described in Figure 1. The alphanumeric designations are described in Table 1. Note the changing ordinate scales for some of the polypeptides.

tial rates of synthesis of 2-50 fold, while five polypeptides responded in a manner similar to the aminoacyl-tRNA synthetases, EF-G and EF-Ts. All polypeptides except F178, showed the most dramatic response (either upward or downward) upon shifting the culture to $42^{\circ} \mathrm{C}$.

From an experiment similar to the one described in Table 1, polypeptide B56.5 was found to have a relative differential rate at $36^{\circ} \mathrm{C}$, approximately 1.5 times that at $28^{\circ} \mathrm{C}(45 \mathrm{~min}$ after the shift), while polypeptide $\mathrm{F} 84.1$ had a rate 3.5 times as fast (data not shown). By integrating the rates of synthesis in the transient period, an accumulation curve can be constructed. Such a curve for polypeptide B56.5 (Figure 4) illustrates the rapidity with which the accelerated rate of synthesis permits the attainment of the new steady state level of this polypeptide: $75 \%$ by $10 \mathrm{~min}$ and $100 \%$ by $15 \mathrm{~min}$ (half-generation) after the shift. This was also true for polypeptide F84.1 (data not shown).

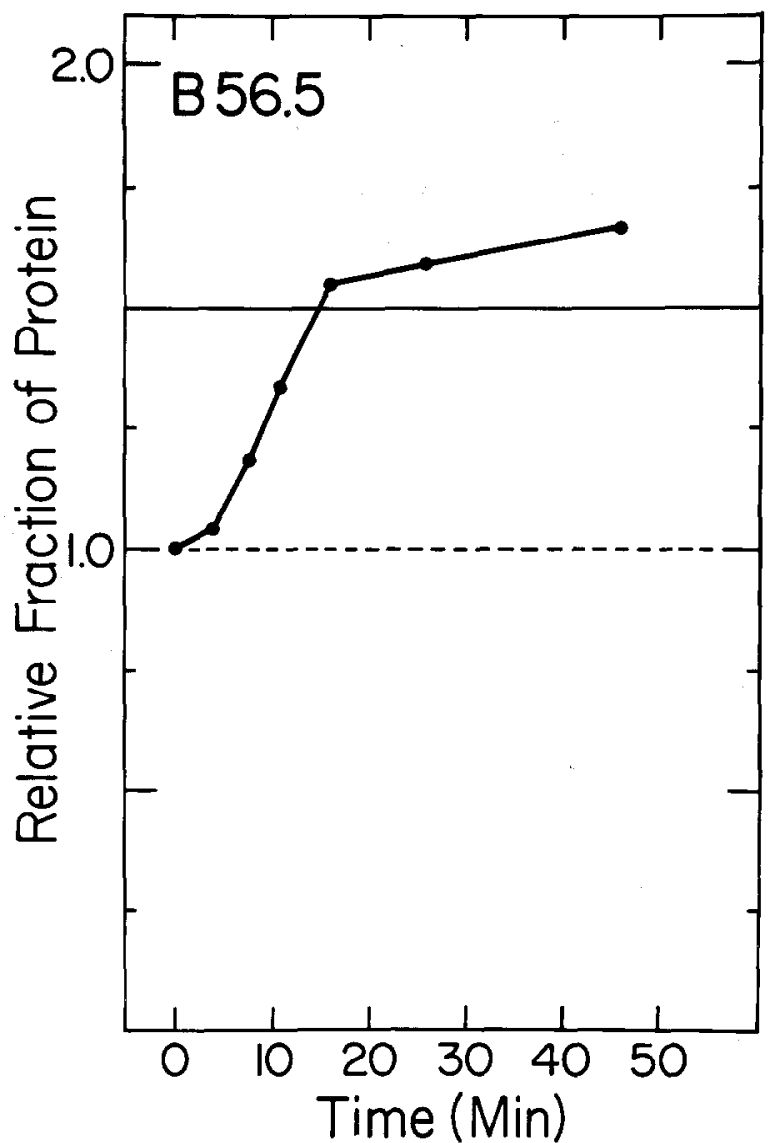

Figure 4. Relative Fraction of Polypeptide B56.5 following a Temperature Shift-up from 28 to $36^{\circ} \mathrm{C}$ in $\mathrm{E}$. coli Strain NC3

The data in this figure were calculated from the relative differential rates of synthesis of polypeptide $B 56.5$ in a temperature shift from 28 to $36^{\circ} \mathrm{C}$. (The experimental data were similar to those depicted in Table 1 , but are not shown.) The relative fraction of B56.5 accumulated at $37^{\circ} \mathrm{C}$ relative to that at $28^{\circ} \mathrm{C}$ was calculated from the following equation:

$F_{p}=A_{i} / \Lambda_{t}+\left(R_{s}\right)\left[1-\left(\Lambda_{i} / A_{i}\right)\right]$

where $F_{p}$ equals the fraction of protein at a given time after the shift to $36^{\circ} \mathrm{C}$ relative to that at $28^{\circ} \mathrm{C}, A_{1}$ equals the initial $A_{420} \mathrm{~nm}$ for the time period of interest, $A_{f}$ equals the final $A_{420} \mathrm{~nm}$ for the time period of interest, and $R_{s}$ equals the average relative differential rate of synthesis for the time period of interest.

The solid horizontal line represents the steady state value for the relative fraction of $B 56.5$ at $37^{\circ} \mathrm{C}$ relative to that at $30^{\circ} \mathrm{C}$.

\section{Effect of a Temperature Shift-down on the Relative Differential Rates of Synthesis of Individual Polypeptides}

The results of a temperature shift from 36 to $28^{\circ} \mathrm{C}$ for six representative polypeptides are shown in Figure 5. For comparative purposes, the data from Figures 1,2 and 3 for the temperature shift from 28 to $36^{\circ} \mathrm{C}$ have been included. The response to a shift-down in temperature was in all cases opposite that of a shift-up. In most cases, not only were the responses in the opposite direction, but the magnitude of the responses was also similar. For example, the relative differential rate of synthesis of polypeptide F84.1 was increased 10 fold by a tem- 


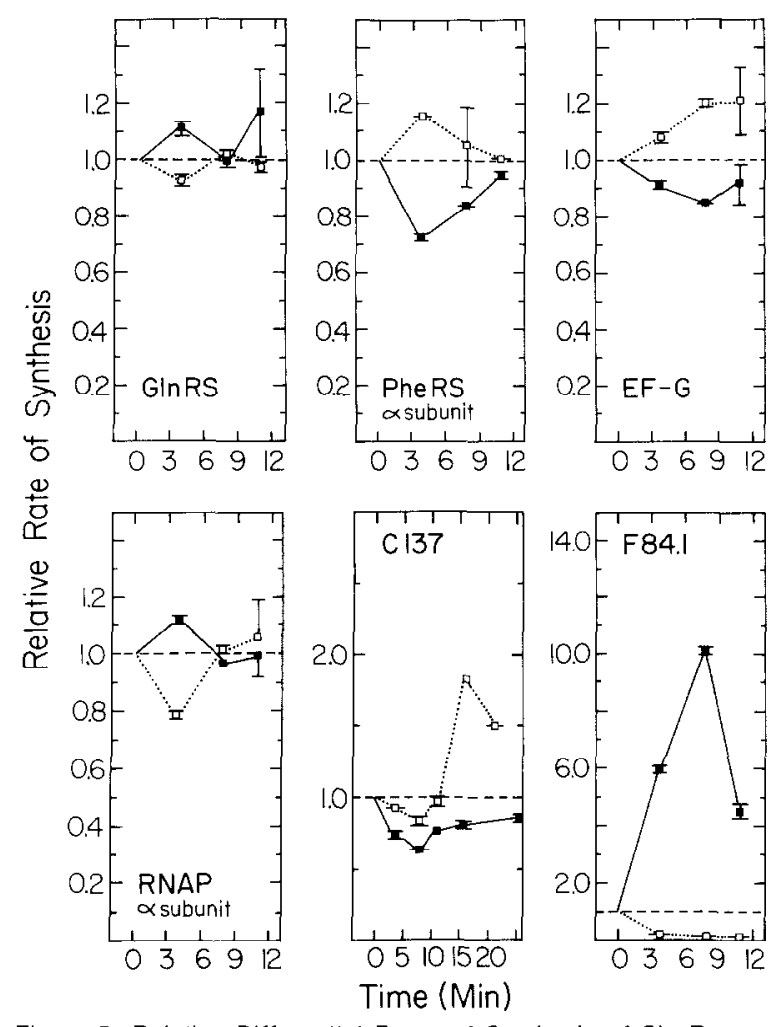

Figure 5. Relative Differential Rates of Synthesis of Six Representative Polypeptides Following a Temperature Shift-down of E. coli Strain NC3

The growth and labeling of cells were as described in Figure 1 and Table 1 , except that the temperature shift was from 36 to $28^{\circ} \mathrm{C}$. Data were calculated as in Table 1 , except that the standard error of the two measurements is included on these graphs as the horizontal bars. Note the changing ordinate and abscissa scales. The open squares (dotted lines) represent the data obtained from the shift from 36 to $28^{\circ} \mathrm{C}$. The data represented by closed squares are replotted for comparative purposes from Figures 1,2 and 3 for the shift from 28 to $36^{\circ} \mathrm{C}$. The abbreviations and alphanumeric designations are as described in Table 1 and Figures 1 and 2.

perature shift-up, while it was decreased to $10 \%$ of its preshift level by a temperature shift-down over the same temperature range.

\section{Production of Guanosine Tetraphosphate upon Temperature Shifts-up of E coli Strain NC3}

Since the response of the translational proteins to temperature shifts are similar to those seen in an amino acid restriction (S. Herendeen, P. G. Lemaux, R. Blumenthal, P. Bloch and F. C. Neidhardt, manuscript in preparation), it seemed possible that ppGpp might have a role in these responses. Figure 6 shows the accumulation of ppGpp following a temperature shift from 28 to $42^{\circ} \mathrm{C}$ using rich medium which lacked isoleucine, leucine and valine (see Experimental Procedures), and the reduced accumulation when this medium was supplemented with these three additional amino acids.

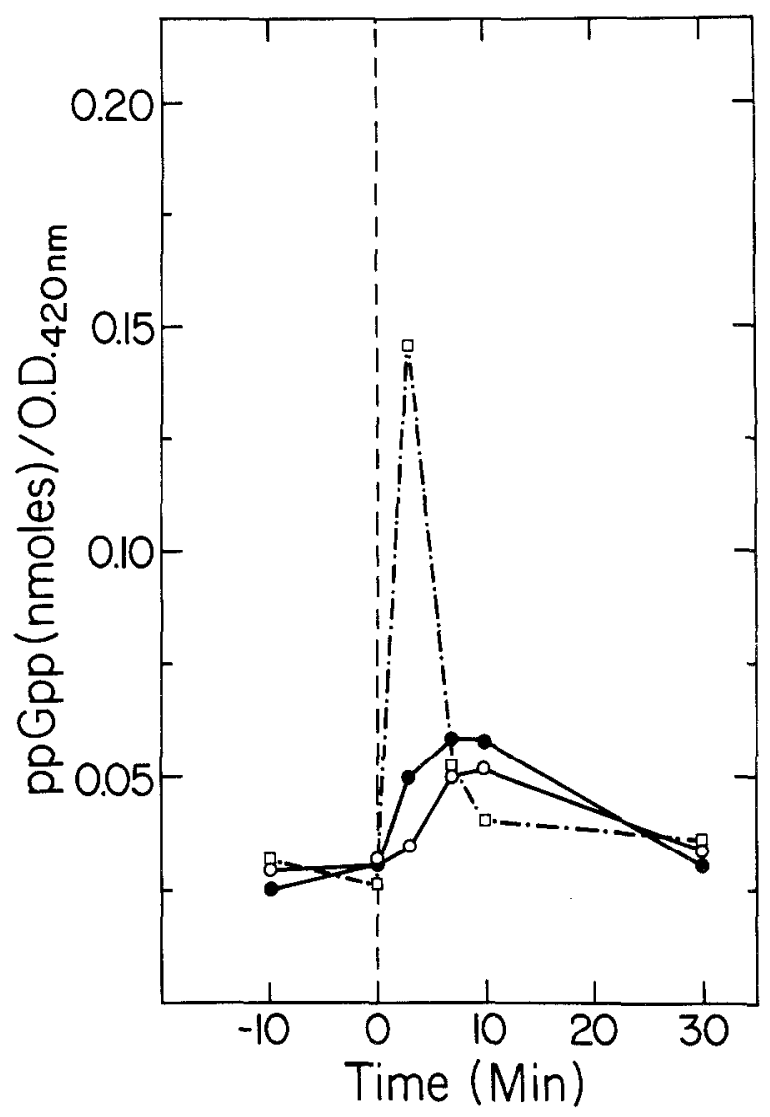

Figure 6. Accumulation of Guanosine Tetraphosphate in E. col Strain NC3 in Several Media upon a Temperature Shift-up from 28 to $42^{\circ} \mathrm{C}$

E. coli strain NC3 was grown up in three different synthetic rich media: one containing all 20 amino acids in "normal" amounts (see Experimental Procedures) (-) ; one containing all 20 amino acids but with the levels of isoleucine, leucine and valine present at 0.1 the "normal" amount ( $\mathrm{O}-\mathrm{O}$ ); and one containing 17 amino acids, lacking isoleucine, leucine and valine $\left(\square \cdot-\square\right.$ ). All media contained $0.132 \mathrm{mM} \mathrm{K}_{2} \mathrm{HPO}_{4}$ sufficient for growth to $\mathrm{OD}_{420 \mathrm{~nm}} 1.0$ and $100 \mu \mathrm{Ci} / \mathrm{ml}^{32} \mathrm{PO}_{4}$. Samples were treated as described in Experimental Procedures. The vertical dashed line represents the time of the shift from 28 to $42^{\circ} \mathrm{C}$.

Representative results for the relative differential rates of synthesis of polypeptides under these two conditions are illustrated in Figure 7 . Of the aminoacyl-tRNA synthetases investigated, only one (valyl-tRNA synthetase) showed a significant difference between the two conditions. The rest of the synthetases showed a response similar to that of phenylalanyl-tRNA synthetase ( $\alpha$ subunit). Compared to the previous conditions, the rates of synthesis of EF-G and ribosomal protein $\mathrm{S} 1$ (and EF-Ts and EF-Tu; data not shown) seemed to be diminished to a lesser extent under conditions where the accumulation of ppGpp was decreased. Among the unidentified polypeptides most, like C137, showed no effect, while a few, like B56.5, showed some difference. A general effect of the 


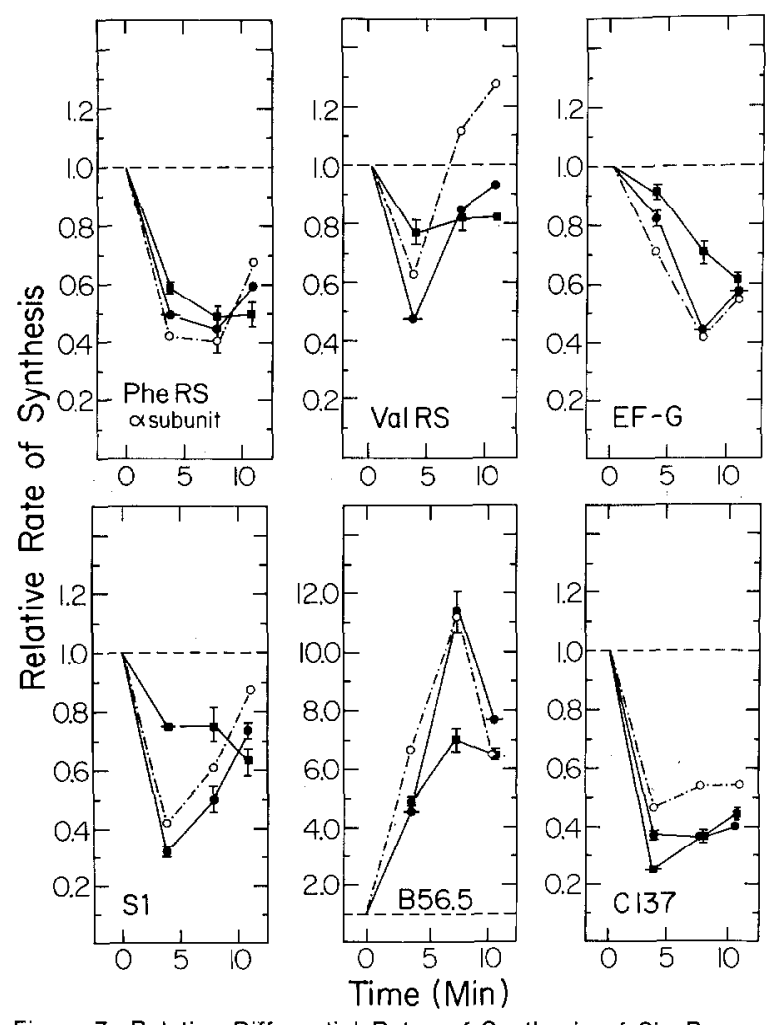

Figure 7. Relative Differential Rates of Synthesis of Six Representative Polypeptides Following a Temperature Shift-up of $\mathrm{E}$. coli Strain NC3 from 28 to $42^{\circ} \mathrm{C}$ in Two Different Media

A culture of $\mathrm{E}$. coli strain $\mathrm{NC3}$ was grown up at $28^{\circ} \mathrm{C}$ in fully synthetic medium lacking isoleucine, leucine and valine. Labeling was as described in Table 1 . Three generations prior to the temperature shift to $42^{\circ} \mathrm{C}$, the culture was split, and 0.1 times the "normal" amount of isoleucine, leucine and valine was added to half. Calculations were performed as described in Table 1 and include the standard error of the two measurements. All abbreviations and alphanumeric designations are as described in Figures 1 and 2 . ( - Data from culture growing in 0.1 the "normal" amount of isoleucine, leucine and valine; $(-)$ ) data from culture growing in medium containing 17 amino acids; and $(0 .-0)$ data reproduced from Figures 1,2 and 3 for the identical shift from 28 to $42^{\circ} \mathrm{C}$ of a culture in medium containing 17 amino acids. This last set of data is reproduced here for comparative purposes.

medium containing the 20 amino acids was to prolong the transient response.

Data collected in an identical experiment (completed 8 months earlier) are included on the graphs in Figure 7 . These data give an indication of the qualitative and quantitative reproducibility of the techniques used.

\section{Discussion}

Since earlier work (Schaechter et al., 1958) had revealed no changes in the overall macromolecule composition of the cell in steady state growth at different temperatures, the transient modulation reported here following temperature shifts was somewhat unexpected. Furthermore, most of the adjustments reported here would not have been discovered by the techniques available until a short time ago. The ability to detect transient changes sensitively in rates of synthesis of individual proteins has been made possible by the combination of dual isotope labeling techniques with the power of the O'Farrell system to resolve complex mixtures of proteins.

Four observations must be accounted for by any model proposed to explain these adjustments in the synthesis of individual polypeptides: first, the rapidity of the changes; second, the return of rates of synthesis of most polypeptides to the preshift rate; third, the direct relation of the magnitude of the response to the size of the temperature shift; and fourth, the opposite and usually equal response to temperature shifts in opposite directions. These transient effects are changes in differential rates of synthesis, and therefore cannot be explained by a general change in protein synthesis rate. For the same reason, they cannot be explained by a direct effect of a restriction in the uptake of the isotopically labeled amino acid.

Because we have measured differential rates of synthesis, it is possible that the effects seen for some polypeptides are passive responses. Intense, specific alterations in the synthesis of one set of polypeptides could change the synthesis rate of other polypeptides since the rates are expressed relative to total protein synthesis. Even so, we are left with the necessity of explaining what signals bring about changes in the rate of synthesis of at least some of the polypeptides upon temperature shift. For example, polypeptide B56.5 seems to exhibit an active, regulatory response to temperature; its synthesis rate responds to a temperature shift, raising the level of this polypeptide to its new steady state value within $15 \mathrm{~min}$. Interestingly, B56.5 is an essential polypeptide, inactivation of which produces cell lysis in a conditional mutant (P. Lemaux and S. Cooper, unpublished results).

What might bring about the active responses? The similarity of the responses of rates of synthesis of the transcriptional and translational polypeptides to a temperature shift-up with their responses to an amino acid restriction (S. Herendeen, P. G. Lemaux, R. Blumenthal, P. Bloch and F. C. Neidhardt, manuscript in preparation) suggested a possible role of the $R e l$ gene and ppGpp. Relevant to this, Gallant, Palmer and Pao (1977) recently found that a temperature shift in a medium lacking certain amino acids caused increased accumulation of ppGpp. By supplementing our medium with isoleucine, leucine and valine, we were able to reduce the increase in $\mathrm{ppGpp}$, upon a temperature shift, to a very low level; yet the responses of the individual polypeptides were essentially the same as those 
observed in the unsupplemented medium. These results, of course, do not exclude the possibility that the small increase in ppGpp in the supplemented medium might be responsible for the transient changes. One means by which a temperature change might produce a cellular signal(s) akin to that produced in nutritional restrictions is by effecting an alteration in the transport of, for instance, an animo acid. This possibility is lent further credence by the observation that temperature affects the transport of $\beta$-galactosides and $\beta$-glucosides (Linden and Fox, 1973). Whatever the nature of such a restriction, it would appear not to involve large changes in ppGpp levels.

It is also possible that changes in rates of synthesis are responses to the activation or inactivation of an enzyme or enzyme system. Particularly inviting areas of metabolism are those responsible for essential functions, such as protein and nucleotide synthesis and energy production, or cellular signals such as cAMP and ppGpp. Patterson and Gillespie (1972) provided evidence for temperature inactivation of initiation factors. Such an inactivation might well generate some signal(s) like amino acid restrictions or nutritional shifts appear to do.

In addition, it is possible that the temperature shifts affect energy production, perhaps even energy charge ratio (Atkinson, 1969). This possibility is suggested by the fact that polypeptide F84.1 may be involved in oxidative metabolism (S. Pedersen, P.L. Bloch, S. Reeh and F.C. Neidhardt, manuscript in preparation). This polypeptide responds in an immediate and dramatic manner to a temperature shift as well as changing its steady state level. Since the energy charge ratio is known to be decreased in cells grown under glucose starvation or anaerobic conditions (Strange, Wade and Dark, 1963), it could be useful to study the effects of a temperature shift on the rates of individual polypeptides in cells such as these.

Another possible effect of a temperature change might involve conformational alterations of, for instance, promoter regions. With respect to this, Travers (1974) has shown that a promoter region exhibits temperature-dependent conformational changes in vitro, and that individual rRNA cistrons may be characterized by multiple promoters, each with a characteristic transition temperature (Travers, 1976). Using this information, it is possible to speculate on the type of promoter structure necessary to give rise to the results obtained in this study.

In summary, the results presented here reveal an array of responses of individual polypeptides to temperature shifts within the normal Arrhenius range of growth (or just outside, to $42^{\circ} \mathrm{C}$ ) of $\mathrm{E}$. coli. These responses are relevant to the general phe- nomenon of cellular adaptation to growth at different temperatures, a naturally occurring event in the life cycle of $E$. coli. Such a phenomenon seems to deserve further study. We would like to investigate further the basis for the common transient behavior of the transcriptional and translational proteins after the shifts, as well as the basis for the dramatic behavior of proteins like B56.5 and F84.1. It would also be of interest to determine whether similar phenomena exist in cells other than $\mathrm{E}$. coli and to extend the current study to other cellular proteins.

\section{Experimental Procedures}

\section{Bacterial Strain}

The $E$. coli $B / r$ derivative NC3 was used in all experiments. Some characteristics of this standard strain have been described (Neidhardt et al., 1977).

\section{Media}

All media used were totally defined synthetic media produced by adding supplements to MOPS (morpholinopropane sulfonic acid)buffered medium (Neidhardt, Bloch and Smith, 1974). Glucose was used as the sole carbon source [final concentration $0.4 \%(\mathrm{~W} /$ v)]. Except where indicated, rich medium was made by adding 17 $\mathrm{L}$ amino acids (lacking isoleucine, leucine and valine for labeling purposes), four nucleic acid bases and five vitamins as previously described (Wanner, Kodaira and Neidhardt, 1977). In the case of the extended time course temperature shifts to 36 or $42^{\circ} \mathrm{C}$, media lacking the nucleic acid bases and vitamins were used. Sterilization of all solutions was by filtration.

\section{Bacterial Growth}

Cultures were grown aerobically at various temperatures (set to within $\pm 0.1^{\circ} \mathrm{C}$ with a thermistor probe) in Erlenmeyer flasks with rotary shaking. Growth rates were determined in cultures between $O_{420} \mathrm{~nm} 0.1$ and 3.0 by suitably diluting the cultures prior to $O D$ measurements. Growth rates are expressed in terms of the specific growth rate constant, $k$, as calculated from the expression $k$ $=(\ln 2) /($ mass doubling time in hr). In experiments involving high levels of radioactivity, growth measurements were carried out on parallel unlabeled cultures. Temperatures shifts were carried out by manually shifting the cultures to a water bath equilibrated at the appropriate temperature, and were completed in less than 15 sec.

\section{Radioactive Labeling}

Proteins were uniformly labeled prior to a temperature shift by growing a culture in rich medium containing radioactive arginine and lysine in amounts sufficient to support growth to $\mathrm{OD}_{420}$ of 1.0 . Cultures were grown from $O D_{420 \mathrm{~nm}}$ of 0.01 to 0.45 in the presence of ${ }^{14} \mathrm{C}$-arginine $(292 \mathrm{mCi} / \mathrm{nmole} ; 10 \mu \mathrm{Ci} / \mathrm{ml})$ and ${ }^{14} \mathrm{C}$-lysine $(318$ $\mathrm{mCi} / \mathrm{mmole} ; 10 \mu \mathrm{Ci} / \mathrm{ml})$ and then chased for $0.5-1.0$ generation with a 50 fold excess of an unlabeled arginine-lysine mixture. To measure the rate of protein synthesis, a $2.0 \mathrm{~m}$ portion of culture was removed and added to a prewarmed flask containing a neutralized labeling mixture of ${ }^{3} \mathrm{H}$-isoleucine $(105 \mathrm{Ci} / \mathrm{mmole} ; 140$ $\mu \mathrm{Ci}),{ }^{3} \mathrm{H}$-leucine $(79.8 \mathrm{Ci} / \mathrm{mmole} ; 225 \mu \mathrm{Ci})$ and ${ }^{3} \mathrm{H}$-valine $(12.5 \mathrm{Ci} /$ mmole; $30 \mu \mathrm{Ci}$ ) which had been dessicated and resuspended in $50 \mu \mathrm{l}$ of medium. Incorporation was permitted for $1.5 \mathrm{~min}$ and then stopped with $44 \mu \mathrm{l}$ of a solution containing unlabeled isoleucine (600 fold excess), leucine ( 570 fold excess) and valine (1000 fold excess). Following further incubation in the presence of the unlabeled amino acids $(1.5-3.5 \mathrm{~min})$, the pulse vials were placed in ice.

\section{Harvesting, Extraction and Electrophoresis}

The harvesting of cells and preparation of cell extracts were 
accomplished in the manner described by Blumenthal, Reeh and Pedersen (1976). A portion of extract $(20 \mu \mathrm{l})$ was applied to the first dimensional isoelectric focusing gel. Second dimensiona SDS polyacrylamide gels $(10 \%)$ were used for the remainder of the procedure as described by O'Farrell (1975). Gels were stained overnight, destained and dried, and autoradiograms were made as described by Pedersen et al. (1976). The identification of the known polypeptide spots has been previously described (O'Farrell, 1975; Furano and Wittel, 1976; Blumenthal et al., 1976; Neidhardt et al., 1977; Reeh, Pedersen and Friesen, 1976).

\section{Measurement of Radioactivity in Gels}

Measurements of the amounts of ${ }^{3} \mathrm{H}$ - and ${ }^{14} \mathrm{C}$-isotope in the twodimensional polyacrylamide gels were made by cutting appropriate portions of the stained gel (aligned with the autoradiogram) with sharpened syringe needles, 15-18 gauge. The samples were individually oxidized in the Packaged Sample Oxidizer as described by Neidhardt et al. (1977) and counted for $10 \mathrm{~min}$. The radioactivity counted was usually well above $100 \mathrm{cpm}$.

\section{Isotope Ratios in Total Protein}

The ${ }^{3} \mathrm{H} /{ }^{14} \mathrm{C}$ ratio fo total protein was determined by absorbing 3 $\mu l$ of cell extract, in quadruplicate, onto a $10 \%$ second dimensional SDS-polyacrylamide gel. The gels were then stained for 3 $\mathrm{hr}$, destained and dried. The entire stained spot was then cut and processed in the Packard Sample Oxidizer.

\section{Measurement of Guanosine Tetraphosphate}

Measurements of ppGpp were performed essentially as described (Cashel, 1969). The phosphate concentration of the MOPS-based medium was reduced to 0.1 of its normal level and was calculated to support growth to $O D_{420 \mathrm{~nm}} 1.0$. At least one generation prior to the temperature shift, portions of the culture were transferred to a flask containing ${ }^{32} \mathrm{PO}_{4}$ to yield a final concentration of $100 \mu \mathrm{Ci}$ / $\mathrm{ml}$. At appropriate times, $50 \mu \mathrm{l}$ of culture were removed into formic acid and spotted in duplicate on thin-layer chromatography plates. The plates were developed, autoradiograms were made and the appropriate spots were cut. The values for ppGpp (nmole) per $\mathrm{OD}_{420 \mathrm{~nm}}$ were then determined. The results were corroborated by using a two-dimensional technique (Gallant, Shell and Bittner, 1976) which lowered the radioactive background.

\section{Chemicals and Radiochemicals}

All chemicals were the highest grade available from ordinary commercial suppliers. Radioactive amino acids and phosphate were purchased from New England Nuclear. The ampholines and other materials used in the polyacrylamide gels were obtained from the suppliers listed by O'Farrell (1975).

\section{Acknowledgments}

This work was supported by grants to F.C.N. from the NSF and from the National Institute of General Medicine Science. This work was performed in partial fulfillment of the requirements of the University of Michigan for the Ph.D. degree of P.G.L., who was supported by a fellowship from the Frederick G. Novy Fund.

The costs of publication of this article were defrayed in part by the payment of page charges. This article must therefore be hereby marked "advertisement" in accordance with 18 U.S.C. Section 1734 solely to indicate this fact.

Received October 12, 1977; revised December 20, 1977

\section{References}

Atkinson, D. E. (1969). Regulation of enzyme function. Ann. Rev. Microbiol. 23; 47-68.

Blumenthal, R. M., Reeh, S. and Pedersen, S. (1976). Regulation of transcription factor $\rho$ and the $\alpha$ subunit of RNA polymerase in Escherichia coli B/r. Proc. Nat. Acad. Sci. USA 73, 2285-2288.

Cashel, M. (1969). The control of ribonucleic acid synthesis in Escherichla coli. J. Biol. Chem. 244, 3133-3141.

Furano, A. V. and Wittel, F. P. (1976). Syntheses of elongation factors $T u$ and $G$ are under stringent control in Escherichia coli J. Biol. Chem. 251, 898-901.

Galiant, J., Shell, L. and Bittner, R. (1976). A novel nucleotide implicated in the response of $E$. coli to energy source downshift. Cell 7, 75-84.

Gallant, J., Palmer, L. and Pao, C. C. (1977). Anomalous synthesis of ppGpp in growing cells. Cell 11, 181-185.

Ingraham, J. L. and Marr, A. G. (1963). Control of enzyme biosynthesis at temperatures near the minimum for growth of Escherichia coli. Coloq. Intern. Centre Nat. Rech. Sci. 124, 319328.

Linden, C. D. and Fox, C. F. (1973). A comparison of characteris tic temperatures for transport in two unsaturated fatty acid auxotrophs of Escherichia coli. J. Supramol. Structure 1, 535-544.

Neidhardt, F. C., Bloch, P. L. and Smith, D. F. (1974). Culture medium for enterobacteria. J. Bacteriol. 119, 736-747.

Neidhardt, F. C., McKeever. W. G. and Parker, J. (1975). Function and regulation of aminoacyl-tRNA synthetases in prokaryotic and eukaryotic cells. Ann. Rev. Microbiol. 29, 215-250.

Neidhardt, F. C., Bloch, P. L., Pedersen, S. and Reeh, S. (1977). Chemical measurement of steady-state levels of ten aminoacyltRNA ribonucleic acid synthetases in Escherichia coli. J. Bacteriol. 129, 378-387.

$\mathrm{Ng}, \mathrm{H}$., Ingraham, J. L. and Marr, A. G. (1962). Damage and derepression in Escherichia coli resulting from growth at low temperatures. J. Bacteriol, 84, 331-339.

O'Farrell, P. H. (1975). High resolution two-dimensional electrophoresis of proteins. J. Biol. Chem. 250, 4007-4021.

Patterson, D. and Gillespie, D. (1972). Effect of elevated temperatures on protein synthesis in Escherichia coll. J. Bacteriol. 112, 1177-1183.

Pedersen, S., Reeh, S., Parker, J., Watson, R. J., Friesen, J. D. and Fiil, N. P. (1976). Analysis of the proteins synthesized in ultraviolet light-irradiated Escherichia coli following infection with the bacteriophage $\lambda d f u s-3$. Mol. Gen. Genet. 144, 339-344. Reeh, S., Pedersen, S. and Friesen, J. D. (1976). Biosynthetic regulation of individual proteins in $\mathrm{re} / \mathrm{A}^{+}$and $\mathrm{rel} A$ strains of Escherichia coli during amino acid starvation. Mol. Gen. Genet. 149, 279-289.

Schaechter, M., Maaløe, O. and Kjeldgaard, N. O. (1958). Dependency on medium and temperature of cell size and chemical composition during balanced growth of Salmonella typhimurium. J. Gen. Microbiol. 19, 592-606.

Strange, R. E., Wade, H. E. and Dark, F. A. (1963). Effect of starvation on adenosine triphosphate concentration in Aerobacter aerogenes. Nature 199, 55-57.

Travers, A. (1974). RNA polymerase-promoter interactions: some general principles. Cell 3, 97-104.

Travers, A. (1976). Multiple modes of ribosomal RNA transcription in vitro. Cell 8, 605-609.

Wanner, B. L., Kodaira, R. and Neidhardt, F. C. (1977). Physiological regulation of a decontrolled lac operon. J. Bacteriol. 130, 212-222. 